E3S Web of Conferences 4, 01001 (2014)

DOI: $10.1051 / \mathrm{e} 3$ sconf/20140401001

(C) Owned by the authors, published by EDP Sciences, 2014

\title{
Phase III (and maybe IV) of the SIMPLE dark matter search experiment at the LSBB
}

\author{
T.A. Girard ${ }^{1, a}$, A.R. Ramos $^{2}$, I.L. Roche ${ }^{3}$ and A.C. Fernandes ${ }^{2}$ (for the SIMPLE Collaboration) \\ ${ }^{1}$ Center for Nuclear Physics and Department of Physics, University of Lisbon, Av. Prof. Gama Pinto 2, \\ 1649-003 Lisbon, Portugal \\ ${ }^{2}$ Campus Tecnológico e Nuclear, Universidade Técnica de Lisboa, EN 10, 2686-953 Sacavém, \\ Portugal \\ ${ }^{3}$ Laboratoire Souterrain à Bas Bruit, Apt 84400, France
}

\begin{abstract}
Phase II, SIMPLE has ended its use of Superheated Droplet Detectors (SDD) in the WIMP search effort. An in-progress Phase III comprises a transition to larger mass bubble chamber technology, using many of the lessons gained from the previous phase since the underlying physics is identical. We describe the progress of the activities, to include several $\sim 1 \mathrm{~kg}$ prototype chambers, each $4 \times$ the total Phase II active mass, which are undergoing testing, prior to a rescaling to a $50 \mathrm{~kg}$ capacity within a Phase IV modular 1 ton detector for a definitive astroparticle search effort.
\end{abstract}

\section{Introduction}

Phase II of the LSBB-sited SIMPLE dark matter search program [1] consisted of two arrays of 15 SDDs each, each operated $\sim 100$ day, which provided internationally competitive results comparable to its North American counterparts PICASSO [2] and COUPP [3] with significantly less exposure. In its duration, Phase II demonstrated the viability of high concentration, relatively inexpensive $\mathrm{C}_{2} \mathrm{ClF}_{5}$ SDD constructions in the search effort $[4,5]$, including the chemistry necessary to achieve detector fabrications with stable operation over extended periods at operating temperatures and pressures capable of providing low energy recoil threshold measurements $[6,7]$. It further demonstrated the ability to construct similar SDDs with various superheated liquids $\left(\mathrm{C}_{3} \mathrm{~F}_{8}, \mathrm{C}_{4} \mathrm{~F}_{10}, \mathrm{C}_{4} \mathrm{~F}_{8}, \mathrm{CF}_{3} \mathrm{I}\right)$ towards eventually providing more restrictive results via different target comparisons [8], and the capacity of a refined, low frequency (LF) acoustic instrumentation to provide discrimination of nuclear recoil events from all gel-associated, environmental, and $\alpha$-induced backgrounds [9, 10].

The difficulty with SDDs for dark matter detection is their low active-to-total mass ratio $(\sim 1-2 \%)$, and that each construct requires 2 day. Phase III comprises a transition to bubble chamber (BC) technology, with a significantly larger mass ratio, enroute to a final Phase IV search experiment with a one ton modular detector.

\footnotetext{
${ }^{a}$ Corresponding author: lcriodets@cii.fc.ul.pt
}

This is an Open Access article distributed under the terms of the Creative Commons Attribution License 4.0, which permits unrestricted use, distribution, and reproduction in any medium, provided the original work is properly cited. 


\section{E3S Web of Conferences}

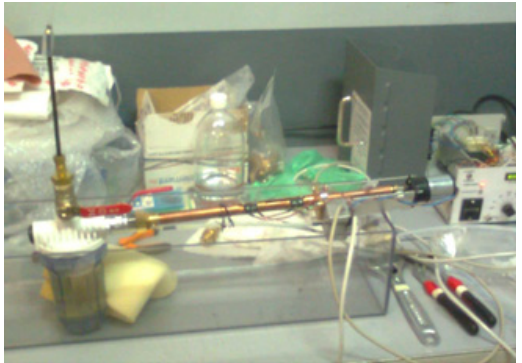

(a)

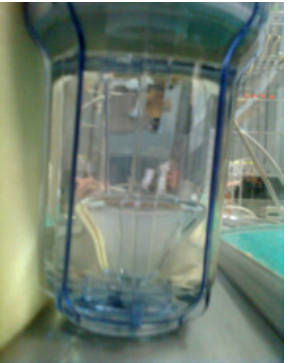

(b)

Figure 1. Prototype $\mathrm{BDC} \mathrm{n}^{\circ} 1(\sim 0.3 \mathrm{~kg}$ active mass) with piston recompression; (b) the "big droplet" within the $\mathrm{BC}$.

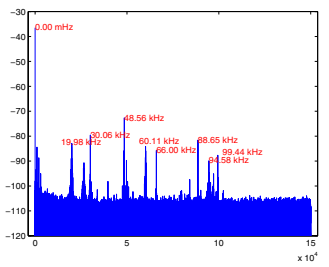

(a)

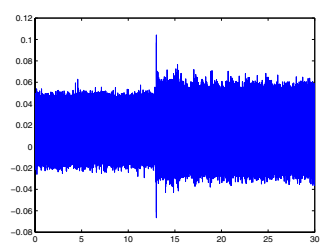

(b)

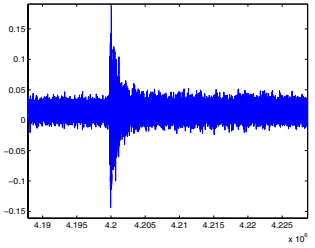

(c)

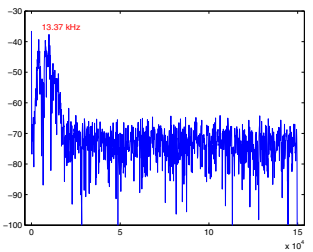

(d)

Figure 2. (a) Prototype HF signal acquisition; (b) correlation with pressure signal: (c) removal of the piston noise by filtering, (d) FFT of the filtered signal in (c). Note that the signal-recompression correlation obviates the need for recording intermediate acoustic data.

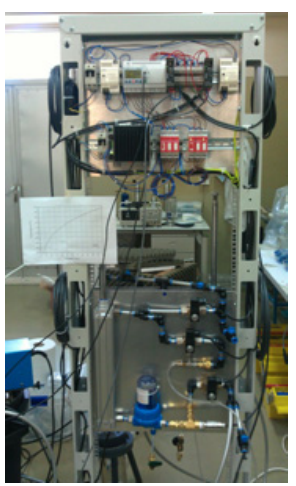

Figure 3. Recompression system.

\section{Phase III}

The BC is essentially a single large droplet SDD, with the same physics base. To avoid the early problems of inhomogeneous nucleation events experienced by COUPP in its developmental phase, the "big droplet" chamber design of Puibasset [11] was resurrected: this consists of a smooth gel sheathing of the inner chamber surface, and creation of a cavity into which glycerol is introduced, followed by the freon. The use of a bulk liquid requires a recompression system capable of rapidly restoring the initial metastable condition of the liquid, preventing its full transition to the gas phase. 


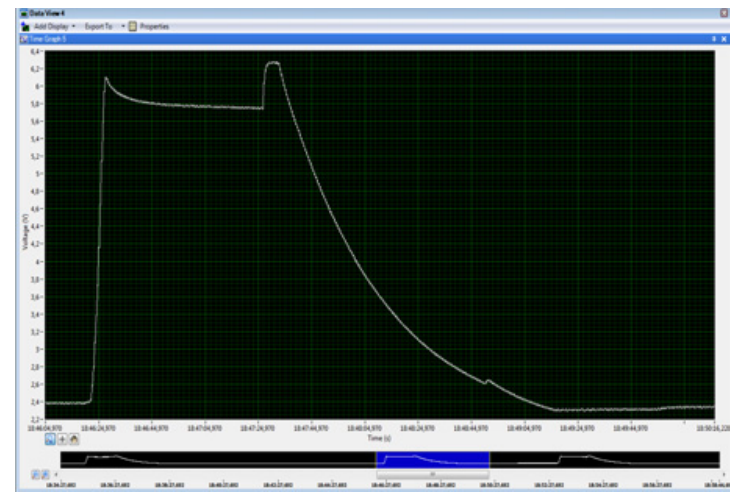

Figure 4. (a) Overall recompression profile, with prototype $\mathrm{BDC} \mathrm{n}^{\circ} 3$ (recomp distance $=5 \mathrm{~m}$, trigger threshold $=100$ mbar).

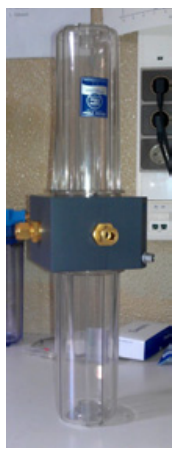

(a)

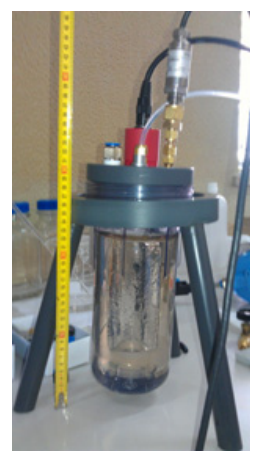

(b)

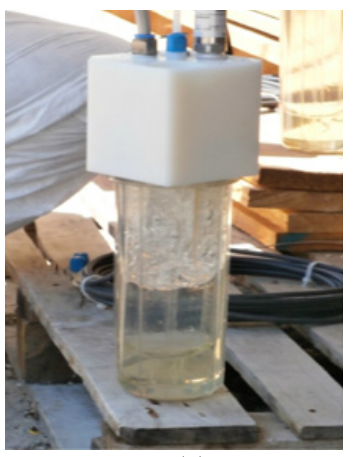

(c)

Figure 5. BC evolution (a) 2-chamber BC for bubble drift studies; (b) mono-chamber with PVC capping, (c) production model with high density PE cap replacement; the PE cap is internally domed to minimize its mass.

Prototyping of the new detector began in 2011, using a low cost "quick \& dirty" home water filter (to permit observation) and $\mathrm{N}_{2}$ re-pressurization, which was quickly replaced by a piston-based system (shown in Fig. 1 during its testing in early 2012) to isolate the freon from the gas. An initial concern of the early testing was the effect of the technique change on the previous particle discrimination capability, which was in part based on the size distribution of the SDD droplets. Given that both PICASSO and COUPP employ high frequency (HF) acoustic instrumentation, signal acquisition was tested with a CM16/CMPA40-5V, 10-250 kHz condenser microphone, and substitution of the current I/O board with the faster NI PCI-6251 1MS/s. As seen in Fig. 2a, although the raw record contained signal events reaching to $100 \mathrm{kHz}$, noise filtering eliminated all but the lower frequencies to leave true nucleation events in a $10-16 \mathrm{kHz}$ window, well within the range of the previous LF system [1]. As seen in Fig. 2b, onset of the piston movement partially masks the event signal, which is recovered by the filtering (Fig. 2c) to yield the FFT of a true nucleation event (Fig. 2d) as observed with the Phase II instrumentation.

Following the 2012 tests, a new "industrial" piston-based recompression system was prototyped, shown completed in Fig. 3. The unit comprises two separate piston subsystems, each capable of driving one BC and both controlled by a single "automaton" based on ladder logic. The overall performance of 


\section{E3S Web of Conferences}

Table 1. Materials' U/Th radio-assays, and background contribution estimates based on the Fig. 6a shield design.

\begin{tabular}{|c|c|c|c|c|c|c|c|c|c|}
\hline & rock & concrete & metal & $\begin{array}{c}\text { water } \\
\text { (tap) }\end{array}$ & $\begin{array}{c}\text { water } \\
\text { (pure) }\end{array}$ & Wood & PE & plastics & paraffin \\
\hline${ }^{232} \mathrm{Th}$ & 0.16 & 7.7 & $1.3 \times 10^{-2}$ & $5.0 \times 10^{-5}$ & $5.0 \times 10^{-5}$ & $3.0 \times 10^{-3}$ & $2 \times 10^{-1}$ & 1.27 & $<0.41$ \\
\hline${ }^{238} \mathrm{U}$ & 5.0 & 10.5 & $3.6 \mathrm{a} 10^{-3}$ & $3.2 \times 10^{-3}$ & $3 \times 10^{-6}$ & $1.1 \times 10^{-1}$ & $4 \times 10^{-1}$ & 2.74 & $<0.25$ \\
\hline Contribution & - & $2.9 \times 10^{-4}$ & -0 & $2.5 \times 10^{-3}$ & $2.6 \times 10^{-5}$ & $6.7 \times 10^{-6}$ & $5.3 \times 10^{-5}$ & $3.2 \times 10^{-3}$ & $8.4 \times 10^{-5}$ \\
\hline
\end{tabular}

Table 2. Plastics radio-assays, and background contribution estimate.

\begin{tabular}{|l|l|l|l|l|l|}
\hline & "SIMONA" & "PET" & "TANK" & "BARRA" & "Acrilico" \\
\hline O origin & (unused) & cap PVC & watertank & shield HDPE & chamber \\
\hline content $(\mathrm{mg} / \mathrm{kg})$ & $0.024 \pm 0.004$ & $<0.35$ & $<0.054$ & $0.032 \pm 0.001$ & $<0.034$ \\
\hline background(evt $(/ \mathrm{kgd})$ & $3.1 \times 10^{-2}$ & $5.3 \times 10^{-1}$ & $8.1 \times 10^{-2}$ & $4.8 \times 10^{-2}$ & $5.0 \times 10^{-2}$ \\
\hline
\end{tabular}

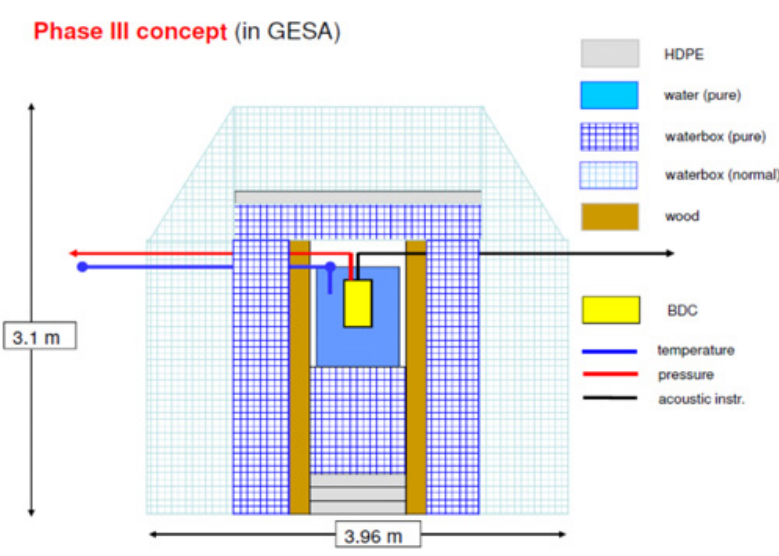

(a)

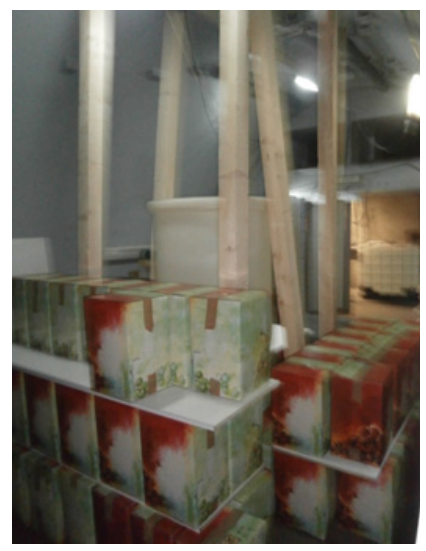

(b)

Figure 6. (a) Schematic of $2 \mathrm{~m}$ thick neutron shield using both normal and purified water, (b) GESA shielding reconstruction (in progress).

the recompression system is shown in Fig. 4, comprising a response time of $2 \mathrm{~s}, 6 \mathrm{~s}$ condensation period, and overall recompression cycle of $163 \mathrm{~s}$.

Testing of the system proceeded throughout 2012-2013, with the BC design evolution shown in Fig. 5. The initial design (Fig. 5a), two centrally-coupled waterfilters with the upper serving as a "drift chamber", was intended to study bubble evolution following observation that it separated from the bulk under its gas buoyancy and rose in the glycerin. Optimization of the recompression timing reduced the drift to within $1 \mathrm{~cm}$ of the bulk droplet, leading to the single filter design of Fig. 5b.

Another concern in the Phase III development was minimization of the background contributions from the U/Th impurities of the experiment construction. Radio-assays of all materials, shown in Table 1, were used to estimate the overall contribution to a measurement based on the preliminary shield design of Fig. 6, which identified the principle contribution to be the containment as in Phase II, and that use of resin-purified water yielded a $10^{2}$ reduction in the background contribution.

A series of assays was made (Table 2) on a variety of plastics, from which the replacement of the PVC by high density PE was suggested given its estimated almost factor 10 lower background contribution $\left(4.8 \times 10^{-2}\right.$ evts $\left./ \mathrm{kgd}\right)$. The final prototype construction shown in Fig. $5 \mathrm{c}$.

In 2013, two chambers and a recompression system were transferred to the LSBB for final testing during the GESA shield reconstruction, with a 30 day preliminary search measurement intended to 


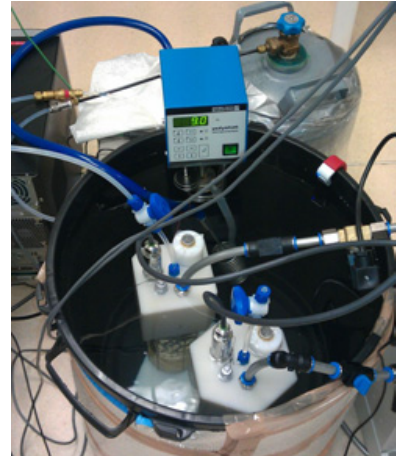

(a)

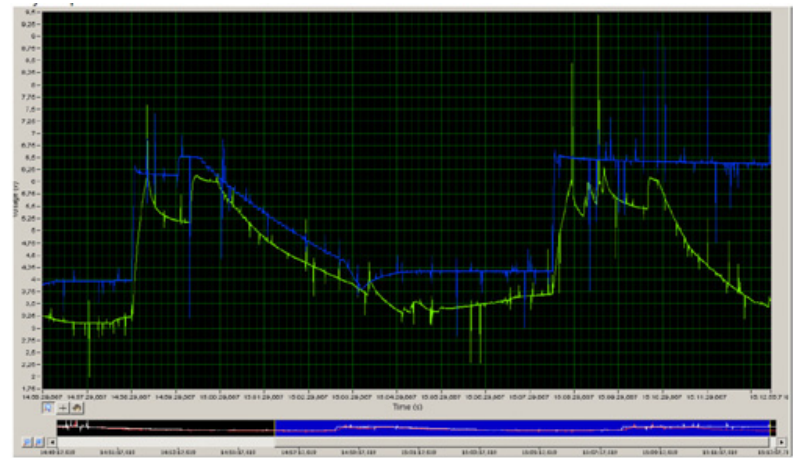

(b)

Figure 7. (a) Two BCs undergoing testing in the LSBB "Salle Blanche"; (b) typical May/June 2013 recompression record, the traces indicating the numerous voltage spikes and inability of the system to return to 2 bar.

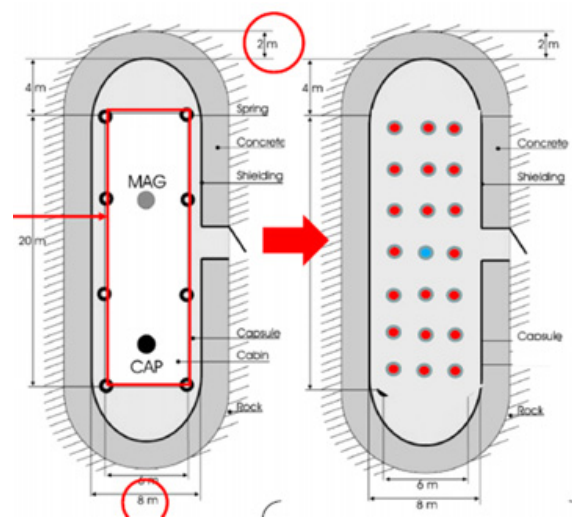

Figure 8. Left: current Capsule configuration, Right: projected SIMPLE reconfiguration, showing the "box" removal and a twenty detector installation in a water-flooded Capsule.

follow. Almost immediately, however, problems developed with the chamber performance, in particular the inability of the recompression system to restore the initial device metastability as seen in Fig. 7.

After 6 weeks of unsuccessful trouble-shooting, the equipment was packed and returned to Lisbon for more detailed diagnostics. The problems have since been identified and rectified, the repressurization problem in fact resulting from an instability in the LSBB "Salle Blanche" power supply, and a return to GESA is imminent.

\section{A possible Phase IV}

To achieve the Phase IV 1 ton detector, the design concept consists of a modular, twenty $\times 50 \mathrm{~kg}$ chamber array, with $2 \mathrm{~m}$ surrounding purified water for neutron shielding. Although the estimated necessary $1200 \mathrm{~m}^{3}$ is large, several underground siting possibilities exist and are currently being explored, including the LSBB, Canfranc Underground Laboratory in the Spanish Pyrénées, and JinPing Laboratory in China (see talk J. Wang, these Proceedings).

Figure 8 shows a possible experiment configuration for siting in the LSBB Capsule, which entails only a removal of the "box" of the former missile launch control, and flooding of the cavern to a depth of 
$4.5 \mathrm{~m}$. Of the 10 recompression systems required, 2 already exist; similarly, all acoustic instrumentation and the temperature control units are in readiness.

\section{Summary}

SIMPLE Phase III has working BCs, with 4 to be installed underground in GESA with a new shielding construct ( $1.5 \mathrm{~m}$ water thickness $4 \pi$, inner 2 ton resin-purified). Expected turn-on now depends on financial support and team strength, both of which have been decimated by current science policies in Portugal.

A SIMPLE Phase IV is being organized, based on a modular array of twenty 50 liter chambers surrounded $4 \pi$ by $2 \mathrm{~m}$ purified water, with an initial objective of a $4 \times 10^{5} \mathrm{kgd}$ exposure (with anticipated significant intermediate results). Apart from funding and underground siting still to be negotiated, the goal implies the need for an overall background level of $\sim 10^{-6} \mathrm{evt} / \mathrm{kgd}$, which as evident from Table 2 will require significant further chamber R\&D to achieve the necessary $10^{4}$ reduction in containment background.

\section{References}

[1] M. Felizardo, T.A. Girard, T. Morlat et al. (The SIMPLE Collaboration): Phys. Rev. D89 (2014) 072013.

[2] S. Archambault, et al.: Phys. Lett. B711 (2012) 153.

[3] E. Behnke, et al.: Phys. Rev. D86 (2012) 052001.

[4] M. Felizardo, et al.: Phys. Rev. Lett. 108 (2012) 201302.

[5] M. Felizardo, T. Morlat, A.C. Fernandes, TA Girard, et al.: Phys. Rev. Lett. 105 (2010) 211301.

[6] T. Morlat, D. Limagne, G. Waysand, et al.: Nucl. Instr. \& Meth. A560 (2006) 339.

[7] M. Felizardo, T. Morlat, T.A. Girard, et al.: Nucl. Instrum. \& Meth. A614 (2010) 278.

[8] M. Felizardo, T. Morlat, J.G. Marques, et al.: Astrop. Physics 49 (2013) 28.

[9] M. Felizardo et al.: Nucl. Instrum. Meth. A589 (2008) 72.

[10] M. Felizardo, R. Martins, T.A. Girard, et al.: Nucl. Instr. \& Meth. A599 (2009) 93.

[11] J. Puibasset, PhD thesis, Université de Paris 7/6 (2000), unpublished. 